\title{
Comparison of the Effectiveness and Comfort Level of Two Commonly Used Mask Ventilation Techniques in a Model
}

\author{
Saqer M Althunayyan, Raied N Alotaibi, Mohammed A Aljanoubi, Musab Z Alharthi, \\ Abdullah M Mubarak, and Ahmed M Al-Otaibi
}

\begin{abstract}
BACKGROUND: Mask ventilation is an important rescue airway skill for providing oxygenation and ventilation. Maintaining a good face mask seal is a fundamental factor for successful ventilation. Therefore, the aim of this study was to compare the effectiveness and comfortability of 2 commonly used mask ventilation techniques. METHODS: A randomized crossover study was performed to compare the 2-handed C-E and 2-handed V-E techniques on a simulation model. Respiratory therapists were recruited by convenience sampling to hold the mask during mechanical ventilation with a fixed tidal volume $\left(V_{T}\right)$ of $500 \mathrm{~mL}$, a rate of 12 breaths $/ \mathrm{min}$ and a PEEP of zero were provided. Each participant performed a 2-min ventilation session, with a total of 24 breaths for each technique. For each technique, we recorded the median $V_{T}$ and the number of successful breaths $(\geq 300 \mathrm{~mL}$ ). Provider comfort was assessed by using a 5-point Likert scale at the end of the 2 techniques. Subgroup analyses were conducted for sex, experience, and height of the participants. RESULTS: Of the 75 respiratory therapists recruited, 74 participants were included in the analysis. There was no statistically significant difference in the median $V_{T}$ between the V-E (417 $\mathrm{mL}$ [interquartile range, 396-427] $\mathrm{mL}$ ) and $\mathrm{C}$-E techniques (410 [interquartile range, 391-423] $\mathrm{mL})(P=.82)$. Approximately $74 \%$ of breaths delivered by the $C-E$ technique were effective, whereas only $68 \%$ of those delivered by the V-E technique were effective $(P<.001)$. Most of the participants reported that using the 2-handed C-E technique was more comfortable. CONCLUSIONS: In our study, the median $V_{T}$ did not differ significantly between the 2 techniques. However, the C-E technique seemed to be superior to the V-E technique in terms of the number of effectively delivered breaths and comfortability. Further studies are recommended for basic airway management techniques. Key words: airway; ventilation; noninvasive ventilation; respiration; difficult mask ventilation; bag-valve-mask; operating room. [Respir Care 2021;66(3):460-465. (C) 2021 Daedalus Enterprises]
\end{abstract}

\section{Introduction}

Mask ventilation is an important clinical skill for airway management; it improves oxygenation and carbon dioxide clearance, and relieves respiratory distress. It is a crucial skill in prehospital emergencies as well as in hospital emergent settings such as ICUs or emergency departments. ${ }^{1}$ In addition, one of the major steps in the operating room is the preoxygenation of patients who are apneic before intubation. ${ }^{2}$ Maintaining a good face mask seal is a fundamental factor for adequate ventilation. Successful mask ventilation requires a patent airway, proper patient positioning, and a sufficient mask seal. Historically, it has been challenging to adequately ventilate a patient because it requires abundant practice to master appropriate head positioning and to achieve the optimal mask grip to minimize air leakage around the seal..$^{3-7}$ Inadequate ventilation could be associated with undesirable patient outcomes, including severe hypoxia and acidosis, which ultimately leads to hypoxic brain injury or even death. ${ }^{8}$

Different techniques and innovations for hand positioning during facial mask ventilation have been induced and investigated over the past few decades. ${ }^{9}$ Three of the most commonly used techniques are the 1-handed C-E technique, 2-handed C-E technique (Fig. 1A), and the modified 2-handed technique V-E technique (Fig. 1B). In the 1-handed C-E technique, the mask is typically held with the left hand and properly placed to cover the bridge of the nose, both malar eminences, and the mandibular alveolar ridge by using the thumb and index fingers, which makes a " $C$ " shape on the mask surface, whereas the little, middle, and ring fingers form an " $\mathrm{E}$ " shape to the left 
of the jaw. ${ }^{7,10}$ For the 2-handed C-E technique, the health care provider thumb and index finger of each hand are placed on the mask surface and form a " $\mathrm{C}$ " shape, and the jaw is lifted toward the mask with the other 3 fingers of both hands, which makes an "E" shape (Fig. 1A). Alternatively, in the 2-handed V-E technique, health care provider uses the thumbs and eminences of both hands to firmly hold the mask, whereas the other fingers are placed behind the angle of the mandible to pull the jaw upward (Fig. 1B). ${ }^{11}$

Over the past few years, there have been several studies that compared the effectiveness of the 3 techniques in adults and children in different settings. ${ }^{11-15}$ In 2014, a manikin study, including 52 medical practitioners and medical students, compared the 1-handed C-E technique and the 2-handed C-E technique. ${ }^{16}$ The study quantified the median expired tidal volume $\left(\mathrm{V}_{\mathrm{T}}\right)$ for each method to assess the efficacy of mask sealing. Compared with the 1handed C-E technique, the 2-handed C-E method resulted in a significantly higher median expired $\mathrm{V}_{\mathrm{T}}$, with a median difference of $56 \%$ and a $95 \%$ CI of $29 \%$ to $65 \%$. In addition, the same study compared the 2-handed C-E and V-E techniques, and it found no significant difference between the 2 methods. ${ }^{16}$ Similar findings were reported by a few studies that included different types of operators, such as physicians, nurses, paramedics, and undergraduate students. ${ }^{14,15,17,18}$

In contrast, some studies found that using the V-E technique to hold the mask was superior to the common C-E technique, particularly in pediatric patients and patients who were obese. ${ }^{13,19}$ In a recent randomized crossover trial, Fei et $\mathrm{al}^{13}$ mostly included nurses,

\footnotetext{
Drs Althunayyan and Alharthi are affiliated with the Department of Accident and Trauma, Prince Sultan Bin Abdulaziz College for Emergency Medical Services, King Saud University, Riyadh, Kingdom of Saudi Arabia. Mr Alotaibi and Mr Mubarak are affiliated with the Department of Basic Science, Prince Sultan Bin Abdulaziz College for Emergency Medical Services, King Saud University, Riyadh, Kingdom of Saudi Arabia. Mr Aljanoubi is affiliated with the Department of Aviation and Marines, Prince Sultan Bin Abdulaziz College for Emergency Medical Services, King Saud University, Riyadh, Kingdom of Saudi Arabia. Dr Al-Otaibi is affiliated with the Department of Emergency Medical Services, Prince Sultan Bin Abdulaziz College for Emergency Medical Services, King Saud University, Riyadh, Kingdom of Saudi Arabia.
}

Mr Alotaibi presented a version of this paper at the SimGHOSTS Saudi event held February 26-28, 2020, in Princess Nourah University, Riyadh, Saudi Arabia.

The authors have disclosed no conflicts of interest.

Correspondence: Raied N Alotaibi MSc, Department of Basic Science, Prince Sultan Bin Abdulaziz College for Emergency Medical Services, King Saud University, Mail box 150019, Riyadh, Kingdom of Saudi Arabia 11474. E-mail: ralotaibi1@KSU.EDU.SA.

DOI: $10.4187 /$ respcare.07949

\section{QUICK LOOK}

\section{Current knowledge}

Mask ventilation is a critical procedure, particularly in a situation in which the patient cannot be intubated. Optimizing an adequate mask seal is one of the techniques that requires mastering and proper training on the optimal technique. The most common hand technique is the C-E technique. The use of the V-E hand technique has been proposed as a superior option.

\section{What this paper contributes to our knowledge}

Among employed respiratory therapists, the use of the 2-handed V-E technique did not result in an increase in the delivered $\mathrm{V}_{\mathrm{T}}$. Further, respiratory therapists delivered a greater number of successful ventilations when using the C-E technique. With regard to the comfortability level, respiratory therapists stated that using the 2-handed C-E technique is more comfortable than using the $\mathrm{V}$-E technique.

physicians, and medical students, and compared the mean expired $\mathrm{V}_{\mathrm{T}}$ of the 2-handed $\mathrm{C}$-E technique with that of the V-E technique in 81 adults who were obese. In Fei et al, ${ }^{13}$ the 2-handed V-E technique was more effective than the $\mathrm{C}-\mathrm{E}$ technique (mean $\mathrm{V}_{\mathrm{T}}, 720 \mathrm{~mL}$ for $\mathrm{V}$-E and $371 \mathrm{~mL}$ for C-E; $P<.001)$. Despite the number of studies conducted to assess the efficiency of the different 2-handed positioning techniques, the findings have been inconsistent. Moreover, none of the previously mentioned studies compared the comfort level for the provider, which may explain why, despite the conflicting evidence with regard to the effectiveness, the most commonly used technique is the C-E technique. ${ }^{20}$

Because most of the previous studies included operators with different levels of clinical training backgrounds, such as physicians, nurses, paramedics, respiratory therapists, and medical students, with different years of experience, we think that this variation in training might be the cause of the inconsistency in the literature. ${ }^{17,21}$ However, other factors with regard to variations in the operator's physical characteristics, such as sex, hand size, and level of experience, have been suggested. ${ }^{16,22}$ Respiratory therapists are accustomed to handling basic airway management and ventilation procedures. Hence, they are considered the benchmark for other health care providers with regard to ventilation techniques. Therefore, in our study, we included only respiratory therapists as participants. Our study aimed to compare the effectiveness and comfortability of respiratory therapist mask ventilation by using the 2-handed C-E and V-E techniques in a standardized simulation environment. 


\section{Simulated Comparison of Two Mask Ventilation Techniques}

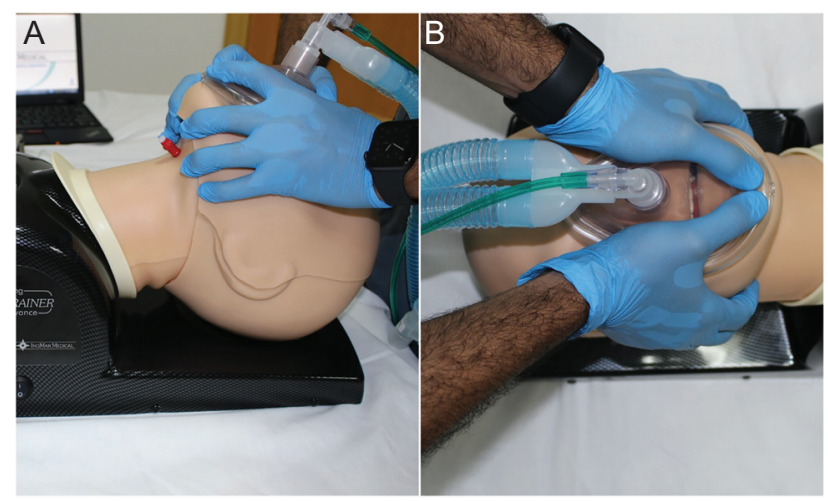

Fig. 1. Two-handed bag-mask ventilation techniques. A: The 2handed C-E technique: the provider's thumbs and index fingers are placed on the mask surface, and the jaw is lifted with the other fingers of both hands. B: The 2-handed V-E technique: the provider uses only his or her thumbs to firmly hold the mask, while the other fingers pull the jaw upward.

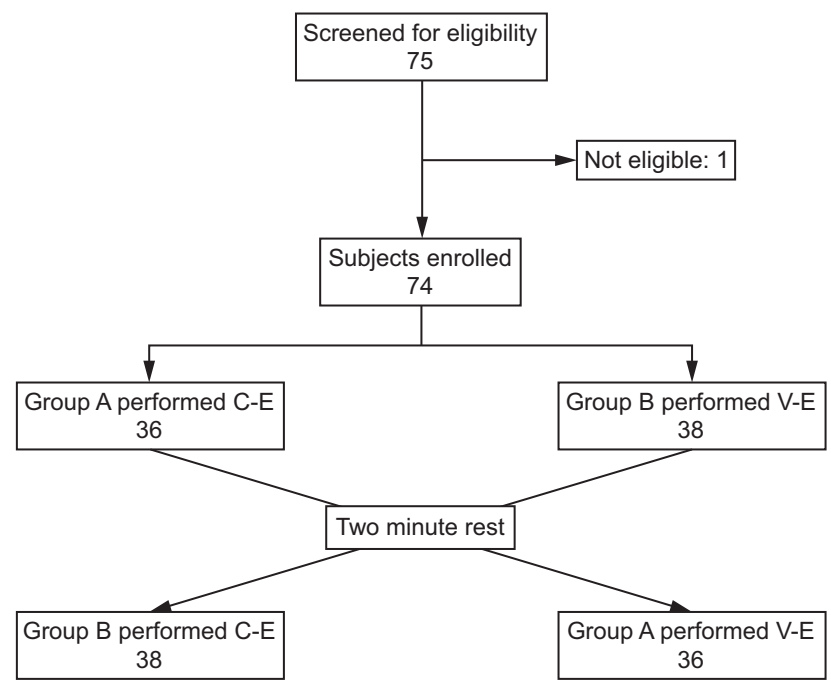

Fig. 2. Flow chart.

\section{Methods}

This study was conducted in the simulation center at Prince Sultan College for Emergency Medical Services. Invitations were sent to respiratory therapists who worked in Riyadh to visit the center. The invitations were sent through social media for respiratory therapists in Riyadh. The eligibility criteria were current work experience and a position as a respiratory therapist doing clinical work in Riyadh. Seventy-five participants joined the study; one was excluded because he worked in administration (Fig. 2). We conducted a pilot study with a group of 20 respiratory therapists at the same center to assess the feasibility of the proposed methodology and to determine the required time to complete the study protocol.
Demographic data were collected by using a set of questionnaires that were self-completed by all the participants. Data on these questionnaires included age, hand dominance, years of experience, department, and other demographics. A randomized crossover design was implemented to achieve the objectives of this study. A simulation-based model was designed in the simulation center at the Prince Sultan Collage for Emergency Medical Services. The model consists of a RespiTrainer advance manikin (IngMar Medical, Pittsburgh, Pennsylvania) and a mechanical ventilator (Puritan Bennett 7200 Mechanical Ventilator; Covidien-Nellcor and Puritan Bennett, Boulder, Colorado), with a constant setting attached to a standard Spur II bagvalve-mask device (Ambu, Ballerup, Denmark) to reduce the variation in $\mathrm{V}_{\mathrm{T}}$ delivered via manual ventilation. The ventilators were programmed in the continuous mandatory ventilation mode to deliver a fixed $\mathrm{V}_{\mathrm{T}}$ of $500 \mathrm{~mL}$ at a rate of 12 breaths/min and PEEP of zero, and the volume that the manikin received was measured. After providing informed consent, the participants were provided with brief instructions on the techniques with a photograph and video describing each technique, and they were asked to demonstrate the 2 techniques on the same manikin. Institutional review board approval was obtained from the ethics research committee at King Khalid University Hospital, King Saud University (research project E-20-4579).

\section{Primary Outcome}

The main outcome of our study was the mean $\mathrm{V}_{\mathrm{T}}$ (in $\mathrm{mL}$ ), which was recorded electronically, and the average value of the measured $\mathrm{V}_{\mathrm{T}}$ for the 2-min sessions for each hand-sealing technique was obtained. Each participant randomly performed a 2-minute session of the 2-handed C-E technique or V-E technique, followed by a rest and then the alternate technique (Fig. 2). The number of breaths with $<300 \mathrm{~mL}$ median $\mathrm{V}_{\mathrm{T}}$ was quantified and classified as ineffective breaths, and breaths with $\geq 300 \mathrm{~mL}$ were considered effective breaths. ${ }^{22}$

Comfortability was assessed through a 4-item, Likertscale questionnaire that consists of 5 points for each question assessing the level of agreement on each question, where 1 indicates that it is very easy to control the mask during the ventilation and 5 indicates that it is too difficult. None of the participants were part of the study team, and all were blinded to the study hypothesis and objectives. In addition, all were blinded to the recorded volumes, and, for all, we muted the alarming system of the ventilator.

\section{Statistical Analysis}

The Shapiro-Wilk test was used to examine the data distribution. Because the data of the primary outcome did not meet the assumption of normality $(P<.01$ for both the C-E 


\section{Simulated Comparison of Two Mask Ventilation Techniques}

Table 1. Characteristics of the Study Participants

\begin{tabular}{lcc}
\hline \hline \multirow{2}{*}{ Characteristic } & \multicolumn{2}{c}{ Ventilation Technique Sequence } \\
\cline { 2 - 3 } & C-E-V-E & V-E-C-E \\
\hline All participants $(N=74)$ & $36(48.6)$ & $38(51.4)$ \\
$\begin{array}{l}\text { Experience } \\
\quad 55 \text { y }\end{array}$ & $17(47.2)$ & $17(44.7)$ \\
$>5$ y & $19(52.8)$ & $21(55.3)$ \\
Sex & & \\
Men & $22(61.1)$ & $20(52.6)$ \\
Women & $14(38.9)$ & $18(47.3)$ \\
Height & & $19(50.0)$ \\
$\leq 165$ cm & $19(52.8)$ & $19(50.0)$ \\
$>165$ cm & $17(47.2)$ & $32(84.2)$ \\
Department & & $1(2.6)$ \\
ICU & $29(80.6)$ & $5(13.2)$ \\
Emergency department & $5(13.8)$ & \\
Others & $2(5.6)$ & \\
\hline Data are shown as $n$ (\%). & & \\
\hline
\end{tabular}

and V-E techniques' median $\mathrm{V}_{\mathrm{T}}$ ), we used a nonparametric Wilcoxon signed-rank test to determine the statistical significance. Subgroup analyses were conducted for sex, height, and years of experience based on the literature findings. Subsequently, in the subgroup analysis that compared 2 independent samples, we used the Mann-Whitney U test. The chi-square test was used to compare categorical variables. Significance was set as $P<.05$. StataCorp 2017, Stata Statistical Software: Release 15.1 (StataCorp, College Station, Texas) was used to perform all the analyses.

\section{Results}

A total of 74 participants, including 42 men and 32 women, were enrolled and performed both techniques. Among the 74 respiratory therapists, 40 participants had $>5 \mathrm{y}$ of experience, and $\sim 82 \%$ were working in an ICU (Table 1). All the participants followed the study protocol without difficulties. The $\mathrm{V}_{\mathrm{T}}$ of 3,552 breaths were measured. The median and interquartile range of $\mathrm{V}_{\mathrm{T}}$ for the $\mathrm{V}-\mathrm{E}$ technique (417 [396-427] mL) was slightly higher than that for the C-E technique (410 [391-423] mL) (Fig. 3). However, the increase was not statistically significant $(P=.82)$.

Moreover, when stratified by participant's physical characteristics, including sex and height, there was no significant difference in the median $\mathrm{V}_{\mathrm{T}}$ between the 2 techniques (Table 2). Approximately $74 \%$ of the breaths delivered by the C-E technique were effective, and, when using the V-E technique, only $\sim 68 \%$ were effective. The number of effective breaths delivered with the $\mathrm{C}-\mathrm{E}$ technique was significantly greater than that delivered with the $\mathrm{V}$-E technique

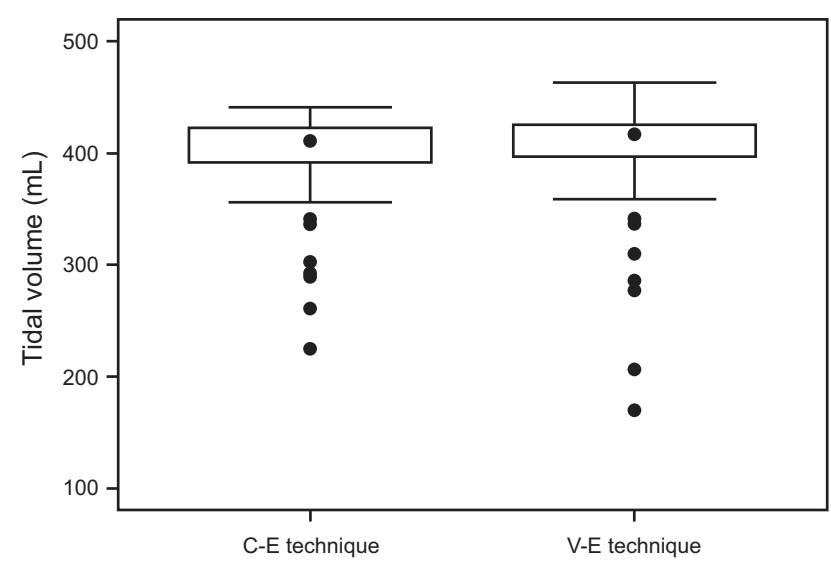

Fig. 3. Box plot of the mean tidal volume when using a mask with the $\mathrm{C}-\mathrm{E}$ and $\mathrm{V}-\mathrm{E}$ techniques. The boxes denote median values, whiskers show $95 \% \mathrm{Cl}$.

Table 2. Median Total $\mathrm{V}_{\mathrm{T}}$ and IQRs Delivered on a Manikin with Both Techniques and the Median Differences for the 74 Participants Overall and Within the Subgroups

\begin{tabular}{|c|c|c|c|}
\hline \multirow{2}{*}{ Outcome Measure } & \multicolumn{2}{|c|}{$\mathrm{V}_{\mathrm{T}}$, Median (IQR) $\mathrm{mL}$} & \multirow{2}{*}{$\begin{array}{c}\text { Median } \\
\text { Difference }\end{array}$} \\
\hline & C-E & V-E & \\
\hline All participants & $410(391-423)$ & $417(396-427)$ & +7 \\
\hline Women & $408(381-422)$ & $407(368-420)$ & -1 \\
\hline Men & $411(394-424)$ & $408(381-422)$ & -3 \\
\hline \multicolumn{4}{|l|}{ Years of experience } \\
\hline$\leq 5 \mathrm{y}$ & $411(395-420)$ & $418(385-423)$ & +7 \\
\hline$>5 \mathrm{y}$ & $409(38-425)$ & $416(399-427)$ & +7 \\
\hline \multicolumn{4}{|l|}{ Height } \\
\hline$\leq 165 \mathrm{~cm}$ & $405(379-422)$ & $412(378-422)$ & +7 \\
\hline$>165 \mathrm{~cm}$ & $416(397-429)$ & $421(406-434)$ & +5 \\
\hline $\begin{array}{l}\mathrm{V}_{\mathrm{T}}=\text { tidal volume } \\
\mathrm{IQR}=\text { interquartile range }\end{array}$ & & & \\
\hline
\end{tabular}

$(P<.001)$ (Fig. 4). Comfortability was assessed by using self-completed questionnaires that were completed after performing both techniques. Among the 74 participants, $40.5 \%$ concluded that V-E was "uncomfortable," and $~ 8 \%$ responded "very uncomfortable." However, only one participant (1.4\%) stated that the C-E technique was "uncomfortable" (Table 3).

\section{Discussion}

In this randomized crossover study, we used an advanced manikin to compare the 2-handed C-E and 2-handed V-E techniques. Seventy-four employed respiratory therapists from different hospitals in Riyadh, Saudi Arabia, were involved, most of whom worked for $>5$ years, which we 


\section{Simulated Comparison of Two Mask Ventilation Techniques}
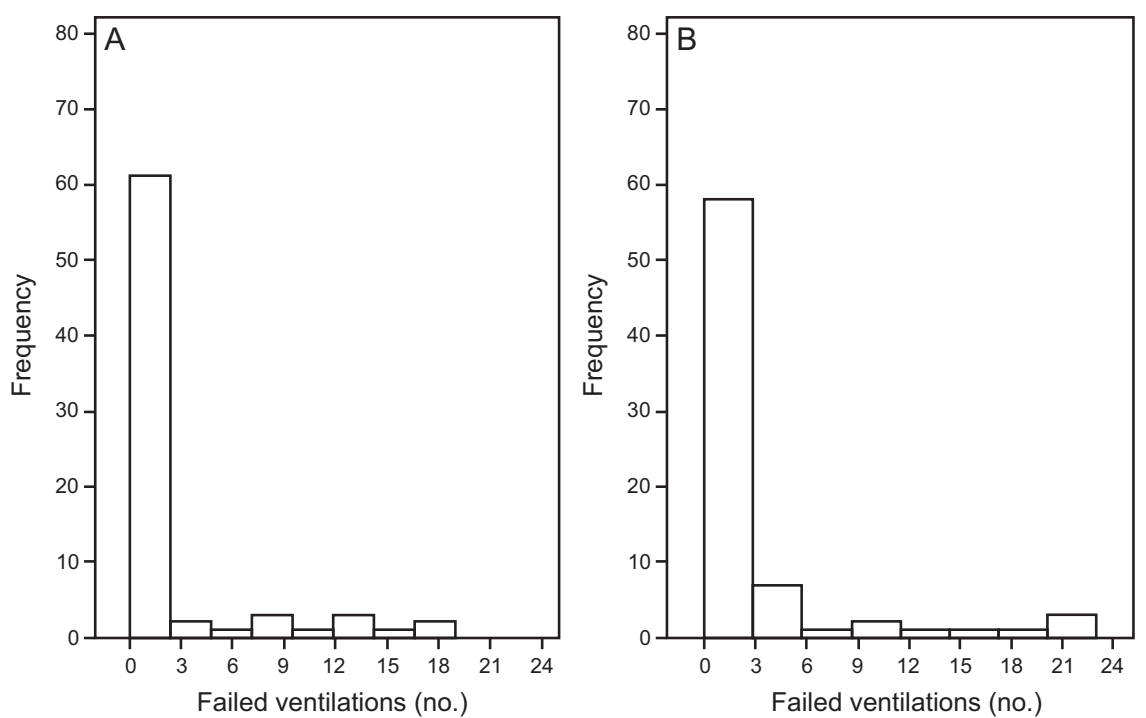

Fig. 4. Distribution of breathing effectiveness ( $\geq 300 \mathrm{~mL}$ is consider effective). Frequency is sorted by the number of failed ventilations when using both techniques. A: The C-E technique, B: The V-E technique.

Table 3. Comparison of the comfortability with Using the C-E and V-E Techniques as Reported by the Participants $(N=74)$ After Performing Both Techniques

\begin{tabular}{lcc}
\hline \hline Comfortability & C-E, $n(\%)$ & V-E, $n(\%)$ \\
\hline Very comfortable & $28(37.8)$ & $10(13.6)$ \\
Comfortable & $33(44.6)$ & $14(18.9)$ \\
Natural & $12(16.2)$ & $14(18.9)$ \\
Uncomfortable & $1(1.4)$ & $30(40.5)$ \\
Very uncomfortable & 0 & $6(8.1)$ \\
\hline
\end{tabular}

believe to be a benchmark in managing basic airway and patients with a difficult airway. We found that there was a mild increase in the median $\mathrm{V}_{\mathrm{T}}$ when using the 2-handed C-E technique compared with the 2-handed V-E technique. However, the magnitude of the increase was not statistically significant. when comparing the number of effective breaths delivered ( $\geq 300 \mathrm{~mL}$ ), the C-E technique was significantly more effective than the V-E technique.

With regard to operator comfortability, we found that most of the participants stated that using the 2-handed C-E technique was more comfortable than using the V-E technique. We believe that the increase in the number of ineffective breaths with the V-E technique could be attributed to the increased number of hand repositioning attempts with the less-comfortable technique. In addition, because fatigue might exaggerate mask leakage, which leads to inadequate ventilation, particularly in prolonged bag-valvemask use situations, this might explain the popularity of the C-E technique among respiratory therapists. ${ }^{20}$

Our subgroup analysis identified that the median $\mathrm{V}_{\mathrm{T}}$ did not significantly vary by sex, height, or years of experience. In contrast, several studies note that females and shorter people usually deliver lower $\mathrm{V}_{\mathrm{T}}$ when ventilating patients who are apneic and when in simulation settings. ${ }^{13,23}$ However, most of the studies included health care providers with different backgrounds, for example, nurses, paramedics, physicians, and medical students. ${ }^{11,18}$ This suggests that the variation in the delivered $\mathrm{V}_{\mathrm{T}}$ is not solely caused by sex or height but that it might be caused by the lack of experience among those health care providers who were not trained as extensively as specialized respiratory therapists; in other words, experience can overcome differences in a provider's physical characteristics.

Our results were consistent with those of Otten et al, ${ }^{16}$ who found no significant difference in the median expired $\mathrm{V}_{\mathrm{T}}$ between the 2-handed techniques. Another study compared the 2 techniques in adults who were apneic and obese, with an average body mass index of $37 \mathrm{~kg} / \mathrm{m}^{2} .{ }^{13}$ The study found that the mask V-E technique is more effective than the C-E technique in ventilating subjects who were obese and concluded that subjects with a difficult airway in whom ventilation failed with the common 2-handed C-E technique could be successfully ventilated with the V-E technique. ${ }^{13}$ However, in our study, we used the same manikin to assess the absolute difference in median $\mathrm{V}_{\mathrm{T}}$, and we standardized the ventilator parameters. Therefore, we believe that if there is a difference between the 2 techniques in terms of the delivered $\mathrm{V}_{\mathrm{T}}$; it is attributable to the effect of the hand-positioning technique not to other related confounders, for example, patient airway anatomy. In clinical situations in which medical procedures might take a long time, such as prolonged bag-valve-mask ventilation, practitioner comfort is always a concern. We recommend developing a more comfortable 


\section{Simulated Comparison of Two Mask Ventilation Techniques}

mask to increase practitioner's comfort level. In addition, we recommend further studies to assess both the efficacy of and comfortability by expert providers with the V-E and C-E techniques in patients with difficult airways.

\section{Limitations}

Several limitations of this study should be emphasized. Due to the nature of the study, it is it is unclear whether the same results would be found in human subjects. We instructed the participants to rest for 2 minutes between the ventilation sessions to avoid fatigue; however, some participants thought they were ready and proceeded without completing the 2-minute rest period. It is unlikely that this limitation would change the results due to the random group allocation of the participants.

\section{Conclusions}

In this simulation-based study, the median $\mathrm{V}_{\mathrm{T}}$ did not change significantly between the 2 techniques. The 2handed C-E technique seemed to be superior to the 2handed V-E technique in terms of the number of effectively delivered breaths and comfortability. Further study to assess both the efficacy and comfortability by expert providers of V-E and C-E techniques in real patients with a difficult airway is recommended.

\section{ACKNOWLEDGMENTS}

The authors thank all the personnel who helped in the data collection: Hatem Altalhi, Mustafa Alhumaidan, and Turki Alharbi. We thank the Simulation Unit at Prince Sultan College for Emergency Medical Services for their cooperation and for providing us with their equipment, despite their busy teaching schedules. The authors thank the Deanship of Scientific Research at King Saud University for supporting this work through research group RG-1441-518.

\section{REFERENCES}

1. Roberts JR, Hedges JR. Roberts and Hedges' clinical procedures in emergency medicine E-book, 6th edition. Philadelphia: Elsevier Health Sciences; 2013.

2. Benumof JL. Preoxygenation best method for both efficacy and efficiency? Anesthesiology 1999;91(3):603-605.

3. Kheterpal S, Martin L, Shanks AM, Tremper KK. Prediction and outcomes of impossible mask ventilation: a review of 50,000 anesthetics. Anesthesiology 2009;110(4):891-897.

4. Adnet F. Difficult mask ventilation: an underestimated aspect of the problem of the difficult airway? Anesthesiology 2000;92(5):1217-1218.
5. Matioc AA. The adult ergonomic face mask concept: historical and theoretical perspectives. J Clin Anesth 2009;21(4):300-304.

6. Orebaugh SL. Difficult airway management in the emergency department. J Emerg Med 2002;22(1):31-48.

7. Saddawi-Konefka D, Hung SL, Kacmarek RM, Jiang Y. Optimizing mask ventilation: literature review and development of a conceptual framework. Respir Care 2015;60(12):1834-1840.

8. Chi JH, Knudson MM, Vassar MJ, McCarthy MC, Shapiro MB, Mallet $\mathrm{S}$, et al. Prehospital hypoxia affects outcome in patients with traumatic brain injury: a prospective multicenter study. J Trauma 2006;61(5):1134-1141.

9. Isono S. One hand, two hands, or no hands for maximizing airway maneuvers? Anesthesiology 2008;109(4):576-577.

10. Brown CA III, ed. The Walls manual of emergency airway management, 5th edItion. Philadelphia: Wolters Kluwer Health; 2017.

11. Strzelecki C, Shelton CL, Cunningham J, Dean C, Naz-Thomas S, Stocking K, et al. A randomised controlled trial of bag-valve-mask teaching techniques. Clin Teach 2020;17(1):41-46.

12. Tracy M, Klimek J, Coughtrey H, Shingde V, Ponnampalam G, Hinder M, et al. Mask leak in one-person mask ventilation compared to two-person in newborn infant manikin study. Arch Dis Child Fetal Neonatal Ed 2011;96(3):F195-F200.

13. Fei M, Blair JL, Rice MJ, Edwards DA, Liang Y, Pilla MA, et al. Comparison of effectiveness of two commonly used two-handed mask ventilation techniques on unconscious apnoeic obese adults. $\mathrm{Br} \mathrm{J}$ Anaesth 2017;118(4):618-624.

14. Joffe AM, Hetzel S, Liew EC. A two-handed jaw-thrust technique is superior to the one-handed "EC-clamp" technique for mask ventilation in the apneic unconscious person. Anesthesiology 2010;113(4):873879.

15. McCabe SM, Smeltzer SC. Comparison of tidal volumes obtained by one-handed and two-handed ventilation techniques. Am J Crit Care 1993;2(6):467-473.

16. Otten D, Liao MM, Wolken R, Douglas IS, Mishra R, Kao A, et al. Comparison of bag-valve-mask hand-sealing techniques in a simulated model. Ann Emerg Med 2014;63(1):6-12.e13.

17. Wood F, Platten C, Byrne S, Wyllie J. Manikin based studies of simulated resuscitation practices: Preterm Face Mask Study. Pediatr Res 2011;70(S5):753-753.

18. Hart D, Reardon R, Ward C, Miner J. Face mask ventilation: a comparison of three techniques. J Emerg Med 2013;44(5):1028-1033.

19. Gerstein NS, Carey MC, Braude DA, Tawil I, Petersen TR, Deriy L, et al. Efficacy of facemask ventilation techniques in novice providers. J Clin Anesth 2013;25(3):193-197.

20. Rajappa GC, Parate LH, Tejesh C, Prathima P. Comparison of modified chin lift technique with EC technique for mask ventilation in adult apneic patients. Anesth Essays Res 2016;10(3):643-648.

21. Walsh K, Cummins F, Keogh J, Shorten G. Effectiveness of mask ventilation performed by hospital doctors in an Irish tertiary referral teaching hospital. Irish Med J 2000;93(2):55-57.

22. Khoury A, De Luca A, Sall FS, Pazart L, Capellier G. Performance of manual ventilation: how to define its efficiency in bench studies? A review of the literature. Anaesthesia 2015;70(8):985-992.

23. Augustine JA, Seidel DR, McCabe JB. Ventilation performance using a self-inflating anesthesia bag: effect of operator characteristics. Am J Emerg Med 1987;5(4):267-270. 